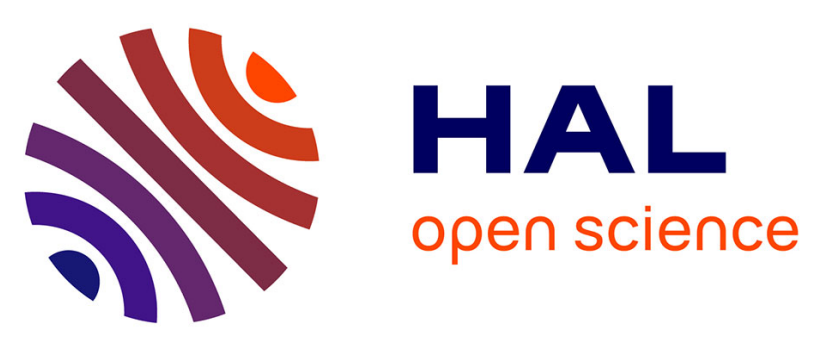

\title{
Absolute 3D reconstruction of thin films topography in microfluidic channels by interference reflection microscopy
}

\author{
A. Huerre, Marie-Caroline Jullien, O. Theodoly, M.-P. Valignat
}

\section{- To cite this version:}

A. Huerre, Marie-Caroline Jullien, O. Theodoly, M.-P. Valignat. Absolute 3D reconstruction of thin films topography in microfluidic channels by interference reflection microscopy. Lab on a Chip, 2016, 16 (5), pp.911-916. 10.1039/c5lc01417d . hal-03152580

\author{
HAL Id: hal-03152580 \\ https://hal.science/hal-03152580
}

Submitted on 25 Feb 2021

HAL is a multi-disciplinary open access archive for the deposit and dissemination of scientific research documents, whether they are published or not. The documents may come from teaching and research institutions in France or abroad, or from public or private research centers.
L'archive ouverte pluridisciplinaire HAL, est destinée au dépôt et à la diffusion de documents scientifiques de niveau recherche, publiés ou non, émanant des établissements d'enseignement et de recherche français ou étrangers, des laboratoires publics ou privés. 


\section{LabonaChip}

\section{Absolute 3D Reconstruction of Thin Films Topography in microfluidic channels by Interference Reflection Microscopy}

\begin{tabular}{|r|l|}
\hline Journal: & Lab on a Chip \\
\hline Manuscript ID & LC-ART-11-2015-001417.R1 \\
\hline Article Type: & Paper \\
\hline Date Submitted by the Author: & n/a \\
\hline Complete List of Authors: & $\begin{array}{l}\text { Huerre, Axel; ESPCI, Gulliver } \\
\text { Jullien, Caroline; ESPCI, Guliver } \\
\text { Theodoly, Olivier; INSERM UMR1067, CNRS UMR7333, AIx Marseille } \\
\text { Université, Adhesion \& Inflammation (U600) } \\
\text { Valignat, Marie-Pierre; INSERM UMR 1067, CNRS UMR7333, Aix Marseille } \\
\text { Université, Adhesion \& Inflammation }\end{array}$ \\
\hline & \multicolumn{2}{|l}{} \\
\hline
\end{tabular}

\section{SCHOLARONE ${ }^{\text {Tw }}$ \\ Manuscripts}




\section{Lab on a Chip}

Miniaturisation for chemistry, physics, biology, materials and bioengineering

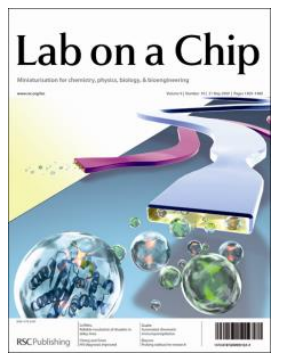

MISSION: To provide a unique forum for the publication of significant fundamental and applied original research related to miniaturisation (on or off chips) at the micro- and nano-scale across a variety of disciplines including: chemistry, biology, bioengineering, physics, electronics, clinical/medical science, chemical engineering and materials science

\section{Impact factor: 6.1 \\ Rejection rate: $70 \%$}

Please consider these high standards when making your decision to accept or reject

\section{Guidelines to Referees}

It is essential that all articles submitted to Lab on a Chip meet the significant novelty criteria; Lack of novelty is sufficient reason for rejection

When preparing your report, please:

- comment on the originality, importance, impact and scientific reliability of the work;

- state unequivocally whether you would like to see the paper accepted or rejected and give detailed comments (with references, as appropriate) that will both help the Editor to make a decision on the paper and the authors to improve it;

- do not make comments about the manuscript or authors which may cause offence.

- do not provide single line reports

When submitting your report, please:

- provide your report rapidly and within the specified deadline, or inform the Editor immediately if you cannot do so;

- submit your report at www.rsc.org/referees

The online service for RSC authors and referees can be found at http://mc.manuscriptcentral.com/rsc

For further information about Lab on a Chip, please visit: www.rsc.org/loc 


\title{
Journal Name
}

\section{ARTICLE}

\section{Absolute 3D Reconstruction of Thin Films Topography in microfluidic channels by Interference Reflection Microscopy}

Received 00th January 20xx Accepted 00th January 20xx

DOI: $10.1039 / \times 0 \times x 00000 x$

www.rsc.org/

\begin{abstract}
A. Huerre ${ }^{a}$, M.-C. Jullien ${ }^{a}$, O. Theodoly ${ }^{b^{*}}$, M.-P. Valignat ${ }^{b} *$
The travel of droplets, bubbles, vesicles, capsules, living cells or small organisms in microchannels is a hallmark in microfluidics applications. A full description of the dynamics of such objects requires a quantitative understanding of the complex hydrodynamic and interfacial interactions between objects and channel walls. In this paper, we present an interferometric method that allows absolute topographic reconstruction of the interspace between an object and channel walls for objects confined in microfluidic channels. Wide field microscopic imaging in reflection interference contrast mode (RICM) is directly performed at the bottom wall of microfluidic chips. Importantly, we show that the reflections at both the lower and upper surface of the microchannel have to be considered in the quantitative analysis of the optical signal. More precisely, the contribution of the reflection at the upper surface is weighted depending on the light coherence length and channel height. Using several wavelengths and illumination apertures, our method allows reconstructing the topography of thin films on channel walls in a range of 0-500 $\mathrm{nm}$, with a precision as accurate as $2 \mathrm{~nm}$ for the thinnest films. A complete description of the protocol is examplified for oil in water droplets travelling in channels of height $10-400 \mu \mathrm{m}$ at a speed up to $5 \mathrm{~mm} \cdot \mathrm{s}^{-1}$.
\end{abstract}

\section{Introduction}

Many microfluidics applications manipulate objects in confined channels where strong interactions occur between objects and channel walls. Typical examples are found in digital microfluidics where microreactors ${ }^{1,2}$ (droplets, vesicles or capsules) or living entities (single cells $^{3-7}$ or small organisms ${ }^{8,9}$ ) are manipulated at high throughput rate in microcircuits. Object-wall interactions play a central role in experimentations with confined items because they strongly influence the shape of the objects, their velocity and the stress that they encounter. Unfortunately, interfacial interactions remain difficult to control and characterise because they result from complex and entangled phenomena, such as the hydrodynamics of thin lubrication films, the deformability of the objects, the composition and rheological properties of the interfaces, or the disjoining pressures between objects and walls. Hence, the characterisation of the contact zone between an object and channel walls is an important issue for both fundamental and applicative reasons, but few studies have investigated these issues especially in microfluidics systems. Reflection interference contrast microscopy (RICM) has proven to be a powerful technique to characterise the thickness of the

\footnotetext{
a. MMN, UMR CNRS Gulliver 7083, PSL research University, ESPCI ParisTech, 10 rue Vauquelin, F-75005 Paris, France.

b. LAI, INSERM UMR S 1067, CNRS UMR 7333,Aix-Marseille Universite 13009 Marseille, France.
}

contact zone between an object and a flat surface ${ }^{10-19}$ in static and dynamic conditions but has hardly been used in microfluidics. RICM is a priori well adapted to perform measurements in microfluidics chips with flat channel walls, which is typically the case of devices microfabricated by conventional photolithography routines ${ }^{7,20}$, specific issues must be considered due to confinement. RICM imaging of objects in microchannels may indeed result from a combination of contributions, mainly the reflection at the lower but also the upper channel surfaces of the channel, the stray signal from the side walls of the channel, as well as the signal refracted by objects edges or diffused by the materials constitutive of the chip. Recovering thickness information from experimental RICM images in microfluidics channels requires therefore specific attention and modelling. In this paper, we present a novel normalisation and analysis method adapted for RICM experimentation in microfluidic channels that takes into account multiple reflections in channels and allows accurate and absolute measurements of thin film thickness at the walls of microfluidic channels.

\section{Principles of interferometric microscopy measurement}

Interferometric measurements are performed here with a wide field microscope in epi-illumination. An image focused on the contact region between an object and the lower wall of a microfluidic channel is taken in RICM, i.e. with a crossed 
polariser/analyser cube and an antiflex objective (Figure 1). Combination of crossed polarisers and quarter wavelength plate in the antiflex objective decreases stray light and increases the signal to noise ratio of images. We used a Zeiss Observer inverted microscope (Carl Zeiss, Jena, Germany) equipped with a Zeiss Neofluar 63/1.25 antiflex objective, a crossed-polarisers cube, a CCD camera (Coolsnap HQ2; Photometrics), and adjustable field and aperture stops. The illumination numerical aperture (INA) was determined for each given aperture opening by measuring the diameter of the illumination cone on a screen positioned at different heights. Accessible INA values were comprised between 0.31 and 0.82 . The source was an X-cite 1200 lamp (Exfo, Mississauga, Canada) coupled to a narrow bandpass filter $(\lambda=546 \mathrm{~nm} \pm 12$ $\mathrm{nm})$. In order to correct for the inhomogeneities of the illumination, raw images were divided by the image of a homogeneous zone (e.g. substrate/solution interface, Figure 2.a) normalised to an average value of 1 . The light intensities $f^{f}$ of these flattened images were then converted into normalised signal $S$ using the intensities measured for the substrate/solution and substrate/chip interfaces. To clarify our purpose, we consider in the following of our discussion the case of microfluidic devices fabricated with Polydimethylsiloxane (PDMS) chips assembled on a glass slide, which corresponds to our experimental conditions. Obviously, the method is not restricted to chips made of PDMS and glass, and for other types of chips, one would just have to consider the correct refractive indices of the materials constituting the chips in the optical modelling. The reference light intensities measured at the glass/water and glass/PDMS interfaces are thereafter called $l^{w}$ and $I^{P D M S}$ and the experimental normalised signal was calculated as:

Eq. 1

$$
S=\frac{I^{f}-I^{P D M S}}{I^{w}-I^{P D M S}}
$$

Data normalisation along Equation 1 allows correction of background noise and of day-to-day variations of source intensity. Moreover, the use of $I^{P D M S}$ and $l^{w}$ as references is convenient for microfluidics because they are directly accessible on the chip with exactly the same optical conditions as for the measurements in channels. The markedly different brightness's of $I^{P D M S}$ and $I^{w}$ is an asset for a good precision of the normalisation procedure. Alternative solutions to get reference signals could be channels filled with different fluids. In the following, I will refer to raw and flattened intensities and $S$ to normalised signals.

\section{Interferometric microscopy measurement between two semi-infinite media}

\subsection{Modelling of optical signal}

In RICM, the total intensity reflected by a sample results from multiple reflections and interferences in the thin layers composing the reflecting interface (light is coherent for thin films with thickness of hundreds of nanometers). With convergent illumination, the total theoretical intensity is obtained by integration over the incident light cone of half aperture $\theta_{0}$ :

Eq. 2

$$
I_{t h 1} \propto \int_{0}^{\theta_{0}} I_{t h 1}\left(\theta_{i}\right) \sin \theta_{i} d \theta_{i}
$$

where $I_{t h 1}\left(\theta_{i}\right)$ is the reflected intensity for light with incidence $\theta_{i}$ calculated by taking into account the Fresnel coefficients of the sample. At the interface between two semi-infinite media $i$ and $\mathrm{j}$ of index $n_{i}$ and $n_{j}$ (Figure 2-a), the parallel and perpendicular Fresnel coefficients are:

Eq. 3

$$
\begin{aligned}
r_{i j}^{p} & =\frac{n_{j} \cos \theta_{i}-n_{i} \cos \theta_{j}}{n_{j} \cos \theta_{i}+n_{i} \cos \theta_{j}} \\
r_{i j}^{S} & =\frac{n_{i} \cos \theta_{i}-n_{j} \cos \theta_{j}}{n_{i} \cos \theta_{i}+n_{j} \cos \theta_{j}}
\end{aligned}
$$

where $\theta_{i}$ and $\theta_{j}$ are the incident and transmitted angles linked by the Descartes-Snell's law:

$$
\text { Eq. } 5 \quad n_{i} \sin \theta_{i}=n_{j} \sin \theta_{j}
$$

and the total intensity $I_{t h 1}\left(\theta_{i}\right)$ is given by ${ }^{18}$ :

Eq. 6

$$
I_{t h 1}\left(\theta_{i}\right) \propto\left|r_{i j}^{s}-r_{i j}^{p}\right|^{2}=R_{i j}
$$

For comparison with experiments, calculated intensities are finally normalised into a theoretical signal $S_{t h}$ using the same normalisation procedure as for experimental intensities:

Eq. 7

$$
S_{t h 1}=\frac{I_{t h 1}-I_{h 1}^{P D M S}}{I_{t h 1}^{W}-I_{t h 1}^{P D M S}}
$$

where $I_{t h 1}, I_{t h 1}^{P D M S}$ and $I_{t h 1}^{W}$ are the theoretical intensities at respectively the glass/fluid ( $n_{i}=n_{\text {glass }}$ and $n_{j}=n_{\text {fluid }}$ ), glass/PDMS $\left(n_{i}=n_{\text {glass }}\right.$ and $\left.n_{j}=n_{P D M S}\right)$ and glass $/$ water $\left(n_{i}=n_{\text {glass }}\right.$ and $n_{j}=$ $n_{\text {water }}$ ) interfaces.

\subsection{Validation of the optical model using glass/water and glass/PDMS interfaces}

We consider the case of a simple interface between a glass plate and an infinite medium (Figure 2-a). Six different media have been tested, namely PDMS elastomer (Sylgard 184 Dow Corning) sealed on a glass substrate, Millipore water, isopropanol (VWR 20839.297), n-hexadecane (Fischer scientific/270/05), mineral oil (SIGMA 8042-47-5) and immersion oil for fluorescence microscopy (Zeiss Immersol $^{\mathrm{TM}}$ $518 \mathrm{~F}$ ). The refractive indices were measured by ellipsometry for PDMS and with a refractometer (Atago PAL-RI) for liquids (see Table 1).

\begin{tabular}{|c|c|c|c|c|c|}
\hline Water & PDMS & Isopro. & Imm. oil & Hexadecane & $\begin{array}{c}\text { Min. } \\
\text { oil }\end{array}$ \\
\hline 1.332 & 1.41 & 1.375 & 1.518 & 1.434 & 1.467 \\
\hline
\end{tabular}

Table 1 Fluids used and their optical indices. Isopro. stands for isopropanol, Imm. Oil for Zeiss immersion oil for microscopy, and min. oil for mineral oil.

RICM images of the glass/media interfaces were taken under monochromatic illumination at a wavelength of $\lambda=546 \pm 5$ $\mathrm{nm}$, with minimal opening of the field stop and two different 
openings of the aperture stop (INA $=0.31$ and 0.82 ). We report on Table 2 the average signals of images normalised using Eq 1 together with the experimental error estimated using Eq. 8:

Eq. 8

$$
\frac{\Delta \mathrm{S}}{S}=\sqrt{\left(\frac{\sqrt{\left(\Delta \mathrm{I}^{\mathrm{f}}\right)^{2}+\left(\Delta \mathrm{I}^{\mathrm{PDMS}}\right)^{2}}}{I^{f}-I^{P D M S}}\right)^{2}+\left(\frac{\sqrt{\left(\Delta I_{w}\right)^{2}+\left(\Delta \mathrm{I}^{\mathrm{PDMS}}\right)^{2}}}{I^{w}-I^{P D M S}}\right)^{2}}
$$

where $\Delta I^{f}, \Delta l^{w}$ and $\Delta I^{P D M S}$ are the experimental quadratic errors of respectively $I^{f}, I^{w}$ and $I^{P D M S}$. Theoretical normalised signals on Table 2 were calculated using Eq 7.

\begin{tabular}{ccccccccc}
\hline Fluid & \multicolumn{2}{c}{ Hexadecane } & \multicolumn{2}{c}{ Isopropanol } & \multicolumn{2}{c}{ Mineral oil } & \multicolumn{2}{c}{ Match oil } \\
\hline INA & 0.82 & 0.31 & 0.82 & 0.31 & 0.82 & 0.31 & 0.82 & 0.31 \\
\hline \multirow{2}{*}{$S$} & -0.16 & -0.12 & 0.38 & 0.37 & -0.37 & -0.39 & -0.47 & -0.49 \\
& \pm 0.04 & \pm 0.02 & \pm 0.04 & \pm 0.04 & \pm 0.04 & \pm 0.03 & \pm 0.04 & \pm 0.03 \\
\hline$S_{\text {th1 }}$ & \multicolumn{2}{c}{-0.2} & \multicolumn{2}{c}{0.37} & \multicolumn{2}{c}{-0.38} & -0.47 \\
\hline
\end{tabular}

Table 2: Experimental and theoretical signals $S$ and $S_{t h 1}$ for different fluids at different INA

The agreement between theoretical and experimental normalised signals for all media and INA conditions is excellent without adjusting parameter. These data therefore validate the correctness of our absolute calculation and the robustness of data normalisation/calibration using intensities at the PDMS and water interfaces with glass.

2.3 Effect of field stop openinglmaging collects reflected signal at the bottom and top contact zone between thr doplet and chanells lower and upper walls.

Figure 3 shows that raw reflected intensities depend markedly on the opening of the field stop, even for a simple interface between two infinite media (here glass/mineral oil). However, the normalised signals using eq. 1 and the $I^{P D M S}$ and $l^{W}$ signals measured at each corresponding field openings are similar for all three conditions of field opening. This shows that the normalisation method corrects efficiently for the effects induced by changes in illumination induced by field stop conditions.

\section{Absolute measurements by interferometric microscopy in closed microfluidic channels}

In this section, we examine the effect of the reflection on the upper wall of a channel on the collected light intensity.

\subsection{Optical model}

We refine here the optical model to take into account the effect of the upper wall (ceiling) of the channel. Let us consider a microfluidic channel of height $H$ (Figure 2-b). The reflecting zone is now composed of two parallel interfaces separated by a distance $H>\lambda$. Light in this thick layer loses its coherence, so that no interference occurs between the beams reflected on the upper and lower interfaces of medium 1 . The resulting reflected signal in the channel is the sum of the intensities of the beam reflected at the $0-1$ interface on one hand and of the beam reflected at the 1-3 interface on the other hand. Since this latter beam crosses twice the $0-1$ interface, it is twice attenuated by the transmission factor $T_{01}=\left(1-R_{01}\right)$. The reflected intensity in a microfluidic channel can finally be written as:

Eq. 9

$$
I_{t h 2}\left(\theta_{i}\right) \propto R_{01}+\left(1-R_{01}\right)^{2} \cdot R_{13}
$$

where the second term represents the contribution of the ceiling reflection. The total intensity for a cone of light with incidence $\theta_{0}$, is given by:

$$
\text { Eq. } 10 \quad I_{t h 2}=\int_{0}^{\theta_{0}} I_{t h 2}\left(\theta_{i}\right) \sin \theta_{i} d \theta_{i}
$$

and the corresponding normalised signal by:

Eq.11

$$
S_{t h 2}=\frac{I_{t h 2}^{f} I_{t h 1}^{P D M S}}{I_{t h 2}^{D}-t_{t h 1}^{D D M S}}
$$

\subsection{Experimental validation of the ceiling reflection contribution}

Let us consider a channel of height $H=25 \mu \mathrm{m}$ with glass substrate and PDMS ceiling filled with a simple fluid (Figure 4b). Table 3 reports the experimental signals $S$ and the theoretical values obtained for different fluids either by not taking $\left(S_{t h 1}\right)$ or by taking $\left(S_{t h 2}\right)$ into account the reflection at the PDMS ceiling.

\begin{tabular}{ccccc}
\hline Fluid & Hexadecane & Isopropanol & Mineral oil & Imm. oil \\
\hline$S_{\text {th1 }}$ & -0.2 & 0.37 & -0.38 & -0.47 \\
\hline$S_{\text {th2 }}$ & -0.13 & 0.33 & -0.2 & 0.02 \\
\hline \multirow{2}{*}{$S$} & -0.1 & 0.31 & -0.23 & -0.09 \\
& \pm 0.06 & \pm 0.09 & \pm 0.08 & \pm 0.07 \\
\hline
\end{tabular}

Table 3: Comparison of the theoretical contrasts for different fluids calculated with a single reflection at the bottom of the channel and with double reflection at bottom and top channel walls. Values correspond to the mean of six different conditions (two INA and three openings of field stop) and the associated error is the quadratic mean of individual errors. Calculations and measurements correspond to a wavelength of $\lambda=$ $546 \mathrm{~nm}$ and a numerical aperture INA $=0.82$ (or INA $=0.31$ that gives the same result for simple interfaces).

It appears clearly in Table 3 that calculations without reflection at the ceiling do not match with experimental data, and conversely that calculations with reflection at the ceiling agree quite well with experimental data. This demonstrates the necessity to consider the reflection across the channel to perform correct RICM measurements in microfluidics.

\subsection{Weighting of the ceiling contribution}

We have checked that the ceiling contribution in RICM signal is significant for channels of height $H=25 \mu \mathrm{m}$ but one expects that this contribution of the ceiling to diminish with channels of larger height and to eventually vanish for very thick channels. In order to establish the range of channel heights where the ceiling contributes to the RICM signal, we 
performed a series of measurements in channels of heights ranging from 10 to $400 \mu \mathrm{m}$ (Figure 4). Channels were filled with mineral oil, INA was fixed at 0.31 , and pictures were taken at three different wavelengths, $\lambda=450 \pm 25 \mathrm{~nm}, 546 \pm$ $10 \mathrm{~nm}$ and $610 \pm 10 \mathrm{~nm}$. In order to assess the contribution of the reflection at the ceiling in the calculations, we introduced a ceiling parameter $p$ as follows :

Eq. 12

$$
I_{t h 3} \propto R_{01}+p\left(1-R_{01}\right)^{2} \cdot R_{13}
$$

where $p$ varies between 0 and 1 , and $p=0$ corresponds to a negligible reflection at the ceiling, whereas $p=1$ to a ceiling reflection that is fully recorded in the experimental image. The adjustable parameter $p$ is determined for each channel height so that theoretical values with Eq. 12 match the experimental data in the corresponding channels. One can see on Figure 4 that $p$ is constant and equal to 1 for channels of height $H$ below $25 \mu \mathrm{m}$. Between $H=25$ and $140 \mu \mathrm{m}, p$ decreases monotonously. Eventually, p vanishes for $H>150 \mu \mathrm{m}$. Ceiling reflection is therefore fully recorded in experimental data for $H$ $<25 \mu \mathrm{m}$, partially recorded for $25<H<150 \mu \mathrm{m}$ and negligible for $H>150 \mu \mathrm{m}$. A correct procedure to perform RICM measurement in microfluidic channel requires therefore an adequate modelling of ceiling reflection but also an empirical determination of the fraction of ceiling reflection that is effectively collected in the conditions of experimental acquisition.

\section{Absolute measurements by interferometric microscopy in closed microfluidic channels containing a droplet}

\subsection{Optical model}

We now consider a droplet squeezed between the upper and lower walls of a channel, with a lubrication film of thickness $d_{1}$ between droplet and walls on both sides (Figure 2-c). Let us first consider the case of a thin film of thickness $d_{1}$ separating the substrate from an infinite medium. The reflecting zone is composed of two parallel interfaces separated by a distance $d_{1}$. The Fresnel coefficients of this stacking made of medium 0 (impinging, semi-infinite), medium 1 (thin film of thickness $d_{1}$ ) and medium 2 (emerging, semi-infinite) can be calculated using the Drude formula ${ }^{21}$ for the $-p$ and the $-s$ polarisation:

$$
\text { Eq. } 13 \quad r_{012}^{s, p}=\frac{r_{01}^{s, p}+r_{12}^{s, p} \cdot e^{-2 i y}}{1+r_{01}^{s, 1} \cdot r_{12}^{s, p} \cdot e^{-2 i y}}
$$

where $y=2 \pi n_{1} d_{1} \cos \theta_{1} / \lambda$ and $\mathrm{I}_{\mathrm{th} 4}\left(\theta_{0}\right)$, the total intensity for a cone of light with incidence $\theta_{0}$, is given by:

Eq.14

$$
I_{\mathrm{th} 4}\left(\theta_{0}\right) \propto\left|r_{012}^{s}-r_{012}^{p}\right|^{2}=R_{012}
$$

For the case of the squeezed object, one has to take into account the reflection on the upper wall of a microfluidic channel of height $H$ (Figure 2-c). The confined object can be considered as a layer of medium 2 with a macroscopic thickness $H$, across which light loses its coherence like in the aforementioned case of a channel filled with a simple fluid. No interference occurs between the beams reflected on the upper and lower interface of medium 2 and the resulting signal reflected in the channel is the sum of the intensities from the beam reflected at the 0-1-2 stacking on one hand and the beam reflected at the 2-1-3 stacking on the other hand. Since this latter beam is also attenuated twice by the transmission factor $T_{012}=\left(1-R_{012}\right)$ due to its double crossing of the 0-12 stacking, the intensity reflected in the channel can finally be written as:

Eq. 15

$$
I_{t h 5}\left(\theta_{0}\right) \propto R_{012}+p\left(1-R_{012}\right)^{2} \cdot R_{213}
$$

\subsection{Example of application}

We now present an application of the method to reconstruct the topography of a water lubrication film separating a moving oil droplet from the walls of a confining microfluidic channel made of a glass substrate bonded to a PDMS chip.

\subsubsection{Experimental}

Glass coverslides used as substrates were cleaned in a Pyranha solutions (Sulfuric acid 95\% / Hydrogen peroxide 50\% - 2/3, $1 / 3 \mathrm{w} / \mathrm{w}$ ) for 5 minutes, rinsed in three different water baths and dried with nitrogen gas. Microchannels were moulded using PDMS (Sylgard 184 Dow Corning) with a $1 / 10 \mathrm{w} / \mathrm{w}$ crosslinker concentration, cured for at least 2 hours at 70 degrees, cleaned with sequential washings with Isopropanol MilliQ water - Decon 5\% - Ethanol - MilliQ Water and dried with nitrogen. Glass coverslips and PDMS chips were finally bonded with an air plasma. Oil-in-water droplets of radius $R=55 \pm 5$ $\mu \mathrm{m}$ were generated at a T-junction in the microfluidic system $^{10,11}$ and driven in a Hele-Shaw cell of height $H=25 \mu \mathrm{m}$. The droplets were composed of mineral oil (SIGMA 8042-47$5)$, whereas the external phase was an aqueous solution of deionized water with Sodium Dodecyl Sulfate (SDS) at 1.42 $\mathrm{mM}$ ( 0.6 times the critical micellar concentration or $\mathrm{CMC}$ ). The surface tension between the two fluids was $\gamma=1.6 .10^{-2} \mathrm{~N} / \mathrm{m}$ at $0.6 \mathrm{CMC}$, and the viscosities were equal to $\mu_{f}=10^{-3}$ Pa.s for the aqueous solution and $\mu_{d}=25.10^{-3}$ Pa.s for the mineral oil. The water flow rate was tuned using a Nemesys syringe pump leading to droplet speed of $5 \mathrm{~mm} \cdot \mathrm{s}^{-1}$. Image of the contact zones between travelling droplets and glass substrate (Figure 6-left) was recorded with a high-speed camera (Photron Fastcam SA4).

\subsubsection{Inversion of a normalised intensity image into absolute thickness map}

Figure 5-a presents the RICM picture of a static oil droplet confined in a $25 \mu \mathrm{m}$ high channel. The contact zone presents an homogeneous brightness indicating an homogeneously flat film. To determine its thickness, we computed theoretical normalized signals for a confined droplet with ceiling coefficient $p=1$, three different wavelengths and two different 
INA values (Figure 5-b). In order to compare theoretical and experimental values, we calculated an error function versus thickness following:

$$
\text { Eq. } 11 \quad \Delta S=\sum_{i=1}^{n} \sum_{j=1}^{m}\left|S-S_{t h 5}\right|_{\left(\lambda_{i}, I N A_{j}\right)}
$$

The minimum of the error function presented in Figure 5-c allows straightforward determination of the best fit between experimental data and theoretical predictions for the 6 couples of $\lambda$ and INA conditions. For the thin film of aqueous solution separating the static drop and channels walls of Figure 5 -a , the best fit on Figure 5-c yields a thickness of $25 \pm 2 \mathrm{~nm}$.

\subsubsection{Topographic reconstruction of a dynamic interface}

The method allows also reconstruction of the topography of thin films with complex and dynamical shapes. Let us consider the contact zone of a droplet of $50 \mu \mathrm{m}$ diameter travelling in a $25 \mu \mathrm{m}$ high channel at a velocity of $500 \mu \mathrm{m} . \mathrm{s}-1$ (Figure 6-a). The reconstructed topography (Figure 6-b) reveals that hydrodynamic effects imprint important structural changes in lubrication film topography. The lubrication film that is perfectly flat for resting drop (Figure 5-a) adopts a complex 3D catamaran-like structure for drop moving at $500 \mu \mathrm{m} . \mathrm{s}^{-1}$. The central zone is roughly $150 \mathrm{~nm}$ thick, whereas the two elongated and narrow side wings are twice thinner. Such analysis procedures on dynamic thin films that yield information with unprecedented in- and out of- plane spatial resolution shall open new avenues in the characterisation and fundamental understanding of the movement of micro-objects in confined geometries.

\section{Conclusion}

We present here a refinement of RICM measurement method that allows absolute measurement of thin films in microfluidic channels. The method takes into account the reflection at the ceiling of channels and allows absolute 3D reconstruction of thin films topography with an accuracy about $\pm 2 \mathrm{~nm}$. For fundamental and applicative perspectives, this method will be of special interest for the dynamics in microchannels of bubbles, droplets, vesicles, capsules, as well as living cells and small organisms.

\section{Acknowledgements}

This work was supported by CNRS, IPGG (Equipex ANR-10EQPX-34), ESPCl, ANR under the contract 13-BS09-0011-01, Labex INFORM and INSERM. 


\section{Figures}

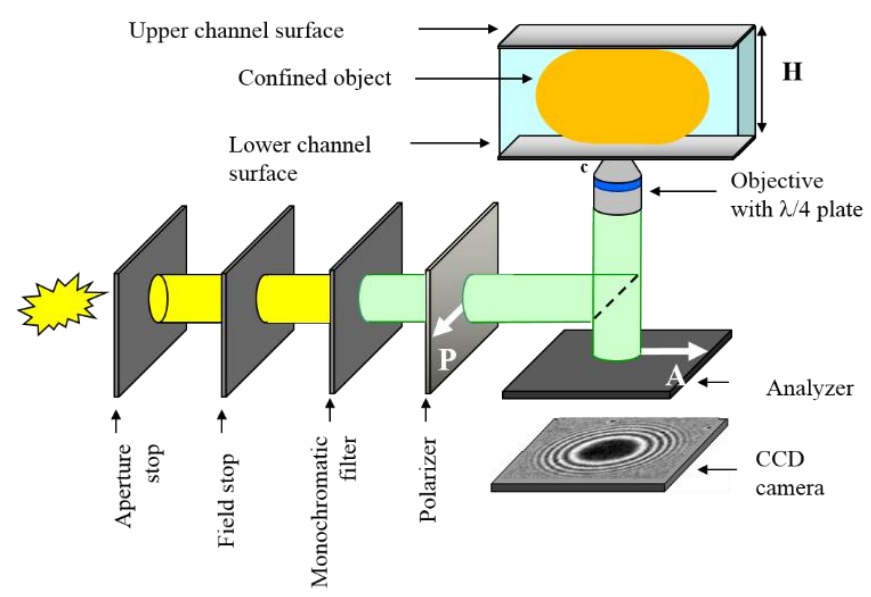

Figure 1 : Schematic of a reflection contrast microscopy (RICM) set up.
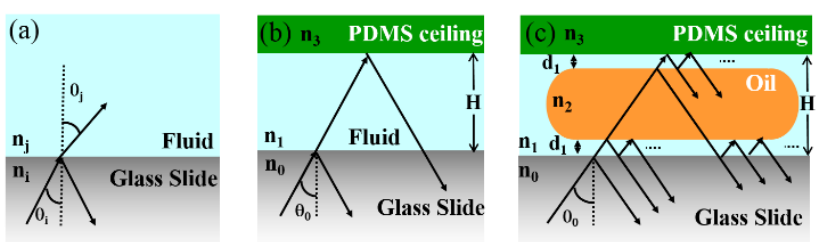

Figure 2: Schematics of the optical configurations corresponding to the systems considerared in our experiments. (a) A simple interface between two infinte medium, a glass substrate and a fluid of known refractive index (b) a microfluidic channel filles with a fluid of known refractiveindex. Imaging collects reflected signal at the bottom glass water and upper water/PDMS interfaces. (c) A confined oil droplet travelling in a microfluidic channel filled with an immiscible fluid. Imaging collects reflected signal at the bottom and top contact zone between thr doplet and chanells lower and upper

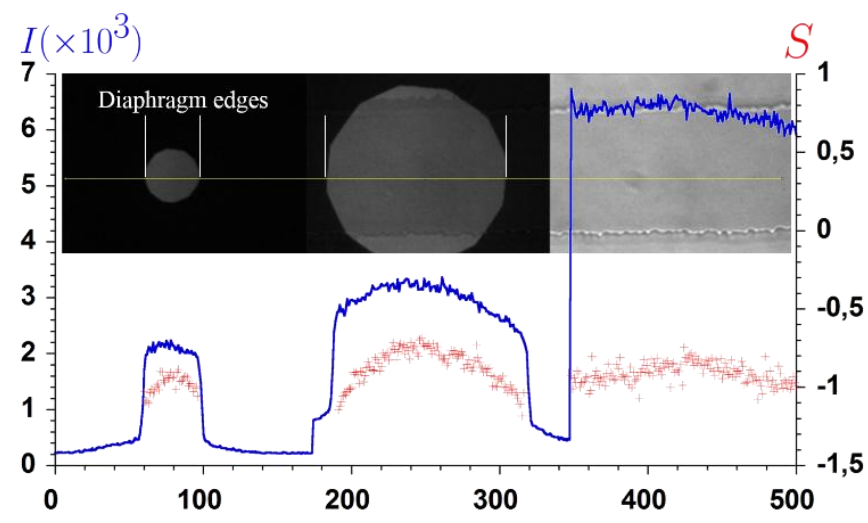

walls.

Figure 3 : Image normalization and illumination conditions. (Top) RICM images of a microfluidic channel filled with mineral oil taken three different openings of the field stop for a focus at the Glass/Oil interface. (Bottom) Profiles of raw intensity (blue line) taken at the centre of images (yellow line in and normalised signal using Equation 1 (red crosses) in the middle of the images.

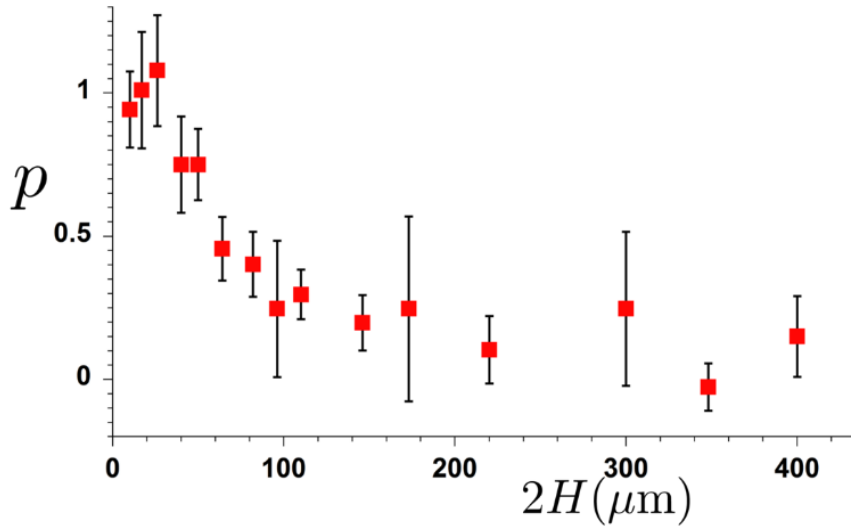

Figure 4 : Contribution of microfluidic channel ceiling to optical reflected signal. Adjustable ceiling parameter $p$ as a function of the channel's height $\mathrm{H}$. Ceiling reflection is fully recorded for $\mathrm{H}<30 \mu \mathrm{m}$ and negligible for $\mathrm{H}>150-200 \mu \mathrm{m}$.

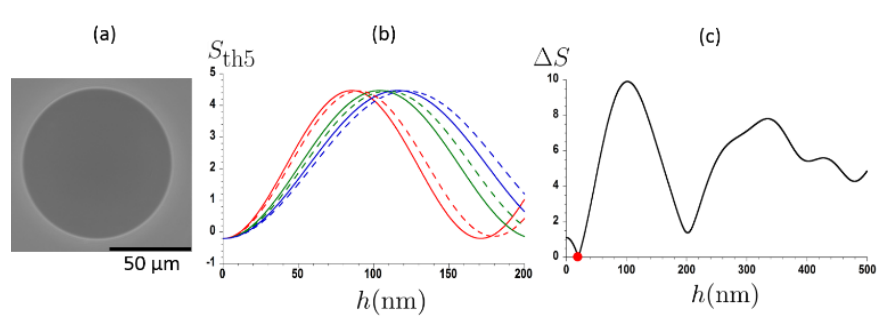

Figure 5 : Conversion of intensity value into absolute thickness. (a) Raw picture of a the contact zone between an oil droplet and the wall of a confining microfluidic channels filled with water. A thin water film separates the oil droplet from the wall . (b) Normalised signal as a function of water film thickness. The dashed curves correspond to an INA of 0.31 , the plain ones to an INA of 0.66 . Red, green and blue colors correspond respectively to wavelengths of 4500,5460 and $6100 \mathrm{~nm}$. (c) Error function $\triangle S$ (Equation 8 ) as a function of the film thickness for one experimental measurement with the 3 wavelengths and the 2 INA. The minimum (red dot) gives access to thickness of the water film.

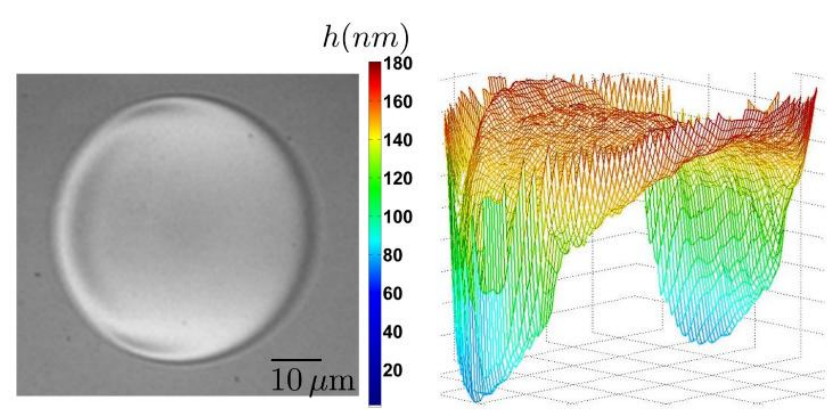

Figure $6: 3 \mathrm{D}$ reconstruction of the topography of the contact zone between a moving droplet and microfluidic channel wall. (Left) Raw image of the droplet contact zone with the lower channel interface of glass. (Right) Reconstruction of the droplet interface using our method of RICM. 


\section{Notes and references}

1 L. Mazutis, J. Gilbert, W. L. Ung, D. A. Weitz, A. D. Griffiths and J. A. Heyman, Nat. Protoc., 2013, 8, 870891.

2 B. Kaoui, T. Kruger and J. Harting, Soft Matter, 2012,

8, 9246-9252.

3S. Gabriele, A.-M. Benoliel, P. Bongrand and O. Theodoly, Biophys. J., 2009, 96, 4308-4318.

4S. J. Tan, L. Yobas, G. Y. H. Lee, C. N. Ong and C. T. Lim, Biomed. Microdevices, 2009, 11, 883-892.

5 M. J. Rosenbluth, W. A. Lam and D. A. Fletcher, Lab Chip, 2008, 8, 1062-1070.

6 P. Preira, V. Grandne, J.-M. Forel, S. Gabriele, M. Camara and O. Theodoly, Lab. Chip, 2013, 13, 161170.

7 R. Hawkins, M. Piel, G. Faure-Andre, A. LennonDumenil, J. Joanny, J. Prost and R. Voituriez, Phys. Rev. Lett., 2009, 102.

8A. San-Miguel and H. Lu, WormBook Online Rev. C Elegans Biol., 2013, 1-19.

9 R. B. Kopito and E. Levine, Lab. Chip, 2014, 14, 764770.

10 A. S. Curtis, J. Cell Biol., 1964, 20, 199-215.

11 D. Gingell and I. Todd, Biophys. J., 1979, 26, 507526.

12 H. Verschueren, J. Cell Sci., 1985, 75, 279-301.

13 J. Radler and E. Sackmann, J. Phys. li, 1993, 3, 727748.

14 G. Wiegand, K. R. Neumaier and E. Sackmann, Appl. Opt., 1998, 37, 6892-6905.

15 I. Weber, Biophotonics Pt B, 2003, 361, 34-47.

16 R. Parthasarathy and J. T. Groves, Biophys. J., 2004, 86, 588A-588A.

17 L. Limozin and K. Sengupta, Chemphyschem, 2009,

10, 2752-2768.

18 O. Theodoly, Z.-H. Huang and M.-P. Valignat, Langmuir ACS J. Surf. Colloids, 2010, 26, 1940-1948.

19 A. Huerre, O. Theodoly, A. M. Leshansky, M.-P. Valignat, I. Cantat and M.-C. Jullien, Phys. Rev. Lett., 2015, 115, 064501.

20 P. Preira, M.-P. Valignat, J. Bico and O. Theodoly, Biomicrofluidics, 2013, 7.

21 G. B. Airy, Philos. Mag., 1833, 20-. 
Confined object travelling in a microfluidic channel

\section{Interference microscopy}

at the object/channel interface

Absolute 3D topography of object in the contact zone 